\title{
Assessing the characteristics and diagnostic value of plaques for patients with acute stroke using high-resolution magnetic resonance imaging
}

\author{
Danfeng Zhang ${ }^{1 \#}$, Mi Wang ${ }^{1 \#}$, Lili Wu ${ }^{2}$, Ying Zhao ${ }^{1}$, Siyu Wang ${ }^{1}$, Xindao Yin ${ }^{1}$, Xinying Wu ${ }^{1}$ \\ ${ }^{1}$ Department of Radiology, Nanjing First Hospital, Nanjing Medical University, Nanjing, China; ${ }^{2}$ Kangda College of Nanjing Medical University, \\ Lianyungang, China
}

Contributions: (I) Conception and design: D Zhang, X Yin; (II) Administrative support: X Yin, X Wu; (III) Provision of study materials or patients: All authors; (IV) Collection and assembly of data: All authors; (V) Data analysis and interpretation: D Zhang, M Wang, Y Zhao, S Wang; (VI) Manuscript writing: All authors; (VII) Final approval of manuscript: All authors.

"These authors contributed equally to this work.

Correspondence to: Xindao Yin; Xinying Wu. Department of Radiology, Nanjing First Hospital, Nanjing Medical University, 68 Changle Road, Nanjing 210006, China. Email: y.163yy@163.com; rebeccahxt@163.com.

Background: A comprehensive understanding of atherosclerotic plaques aids physicians in evaluation and treatment of stroke. This study set out to evaluate the characteristics and diagnostic value of atherosclerotic plaques in patients with acute stroke and stenotic middle cerebral artery (MCA) using high-resolution magnetic resonance imaging.

Methods: Sixty-five consecutive patients with transient ischemic attack or recent ischemic stroke were prospectively recruited. All enrolled patients underwent routine magnetic resonance scans and crosssectional scans of the stenotic MCA vascular wall. Differences in vascular wall parameters and location, the enhancement degree, and remodelling patterns of plaques in the stenotic MCA were compared between symptomatic $(n=30)$ and asymptomatic $(n=35)$ groups of patients. The statistically significant indicators were then subjected to logistic regression analysis to identify which factors could better predict acute stroke.

Results: Compared with the asymptomatic group, the symptomatic group had a smaller lumen area (LA) $(\mathrm{P}=0.027)$, larger plaque area $(\mathrm{P}<0.001)$, larger remodelling index $(\mathrm{P}<0.001)$, more superior/posterior plaques $(\mathrm{P}=0.001)$, more obviously enhanced plaques $(\mathrm{P}<0.001)$, and a greater number of $\mathrm{PR}$ patterns $(\mathrm{P}<0.001)$ in the stenotic MCA. Logistic regression analysis showed that the plaque area, remodelling patterns, LA in the stenotic MCA, enhancement degree, and plaque location were predictors of acute stroke. The combination of the plaque area and LA in the stenotic MCA, and the plaque enhancement degree had optimal predictive value (area under the curve $=0.927$ ).

Conclusions: A larger plaque area and smaller LA in the stenotic MCA, and obvious plaque enhancement might indicate that a patient is prone to acute stroke.

Keywords: Magnetic resonance imaging; middle cerebral artery (MCA); atherosclerosis; vascular remodelling

Submitted May 16, 2021. Accepted for publication Oct 19, 2021.

doi: $10.21037 /$ qims-21-531

View this article at: https://dx.doi.org/10.21037/qims-21-531 


\section{Introduction}

Intracranial atherosclerotic disease (ICAD) is a major cause of ischemic stroke globally $(1,2)$. Acute stroke resulting from ICAD is more prevalent in East Asians than in Westerners (3). The middle cerebral artery (MCA) is the most common vessel affected by atherosclerosis in Asian patients (4). Stroke can result in death, a poor quality of life, and a heavy economic burden for patients and their families (5). Therefore, determining the mechanism and predictors of acute stroke is crucial.

In recent years, the focus of a rising number of studies and scholars has shifted to the vessel wall, in particular the features and stability of atherosclerotic plaques from the stenotic degree of intracranial arteries (6-10). Meanwhile, high-resolution magnetic resonance imaging (HRMRI) has become a widely used non-invasive method for depicting the characteristics of plaques and stenotic vessel walls (11-13).

Common features of atherosclerotic plaques include remodelling, enhancement, and plaque burden, among others. Previous studies $(1,8)$ have presented two types of remodelling patterns in arteries: positive remodelling (PR) and negative remodelling (NR). NR contributes to lumen constriction, while $\mathrm{PR}$ causes vessel expansion and alleviates the degree of vessel stenosis. Previous studies $(1,14)$ have reported that $\mathrm{PR}$ is related to acute stroke. Other studies have suggested that obviously enhanced plaques are associated with recent ischemic events $(13,15,16)$. Research has also found that patients with MCA-induced stroke recurrence have a higher plaque burden (11). Furthermore, a handful of studies have explored the relationship between plaque composition and stroke $(2,7)$.

A sufficient understanding of the characteristics and diagnostic value of atherosclerotic plaques aids physicians in evaluating the risk of stroke and designing optimal personalised treatment. However, the abovementioned studies were limited, and few investigations have focused on the association between the plaque features and acute stroke. Therefore, the current study aimed to comprehensively evaluate the characteristics and diagnostic value of atherosclerotic plaques in patients with acute stroke and MCA stenosis using HRMRI.

The following article is presented in accordance with the STAndards for the Reporting of Diagnostic accuracy studies (STARD) checklist (available at https://dx.doi. org/10.21037/qims-21-531).

\section{Methods}

\section{Study participants and clinical data}

From January 2016 to May 2020, 65 consecutive patients from the Department of Neurology (Nanjing First Hospital) were recruited in line with the following inclusion criteria: (I) two or more atherosclerotic risk factors; (II) single MCA M1 segment stenosis (30-99\%) on magnetic resonance (MR) angiography; (III) the degree of stenosis in the ipsilateral internal carotid artery did not exceed 50\%; (IV) clear MR images for diagnosis and analysis. Exclusion criteria: (I) non-atherosclerotic vasculopathy, such as cerebral haemorrhage, vasculitis, moyamoya disease, dissection, or tumour; (II) arterial fibrillation or cardioembolism; (III) contraindications for MR scanning.

This study was conducted in accordance with the Declaration of Helsinki (as revised in 2013). The ethics committee of Nanjing Medical University approved the research protocol. All enrolled patients gave written or verbal informed consent to participate in the study.

Patients' clinical characteristics and demographic details were collected from their medical records. The data collected included age, sex, alcoholism (drinking $>2 \mathrm{U}$ per day on average for men or $>1 \mathrm{U}$ per day on average for women over the past year), diabetes (defined as treatment with antidiabetic medications or fasting plasma glucose $\geq 7.0 \mathrm{mmol} / \mathrm{L}$ ), smoking (smoking $\geq 1$ cigarette a day for 6 continuous months), hypertension (defined as diastolic blood pressure $\geq 90 \mathrm{mmHg}$, systolic blood pressure $\geq 140 \mathrm{mmHg}$, or ongoing treatment with antihypertensive drugs), and the values of haemoglobin A1c (HbA1c), blood glucose, low-density lipoprotein (LDL), high-density lipoprotein (HDL), phospholipase-A2, triglycerides, total cholesterol, homocysteine, and white blood cell count. Patients who had smoked in the past or were current smokers were considered to have a smoking history.

\section{MR protocol}

One week after admission, the enrolled patients underwent MR imaging using routine protocols, including axial plain scan diffusion-weighted imaging (DWI), T1-weighted imaging (T1WI), T2-weighted imaging (T2WI), T2 fluid-attenuated inversion recovery, and enhanced MR angiography. HRMRI scanning of the stenotic MCA was performed using a 3 Tesla MR scanner equipped with an 
8-channel head coil (Ingenia, Philips Medical Systems, The Netherlands).

Maximum intensity projection was reconstructed from the enhanced MR angiography images was used to locate the stenotic site of the MCA. Next, black-blood precontrast T1WI, proton density-weighted imaging (PDWI), and contrast-enhanced T1WI $(\mathrm{T} 1 \mathrm{WI}+\mathrm{C})$ perpendicular to the M1 segment of the MCA were performed, with the scan parameters set as follows: (I) enhanced MR angiography: echo time (TE), $3.45 \mathrm{~ms}$; repetition time (TR), $22 \mathrm{~ms}$; matrix size, $332 \times 227$; number of excitation (NEX), 1; field of view (FOV), $200 \mathrm{~mm} \times 84 \mathrm{~mm}$; slice thickness, $0.6 \mathrm{~mm}$; slice number, 140; acquisition time, $62 \mathrm{~s}$; (II) T1WI: TE, $9 \mathrm{~ms}$; TR, 1,000 ms; slice thickness, $2.0 \mathrm{~mm}$; slice gap, $0 \mathrm{~mm}$; and slice number, 6; NEX, 2; matrix size, $180 \mathrm{~mm} \times 144 \mathrm{~mm}$; FOV, $80 \mathrm{~mm} \times 80 \mathrm{~mm}$; acquisition time, $192 \mathrm{~s}$; (III) PDWI: TE, $9 \mathrm{~ms}$; TR, 2,000 ms; slice thickness, $2.0 \mathrm{~mm}$; slice gap, $0 \mathrm{~mm}$; and slice number, 6; NEX, 2; matrix size, $180 \mathrm{~mm} \times 144 \mathrm{~mm}$; FOV, $80 \mathrm{~mm} \times 80 \mathrm{~mm}$; acquisition time, $150 \mathrm{~s}$; and (IV) T1WI+C: TE, $9 \mathrm{~ms}$; TR, 1,000 ms; slice thickness, $2.0 \mathrm{~mm}$; slice gap, $0 \mathrm{~mm}$; and slice number, 6; NEX, 2; matrix size, $180 \mathrm{~mm} \times 144 \mathrm{~mm}$; FOV, $80 \mathrm{~mm} \times 80 \mathrm{~mm}$; and acquisition time, $192 \mathrm{~s}$. A bolus of $15 \mathrm{~mL}$ of gadodiamide (Kang Chen, Guangzhou, China) was delivered via intravenous injection at a rate of $2 \mathrm{~mL} / \mathrm{s}$, and $\mathrm{T} 1 \mathrm{WI}+\mathrm{C}$ was performed 5 minutes after the injection.

\section{MR image analysis}

All images must be clear for diagnosis and analysis. The Philips Intellispace Portal workstation was used for measurement of vessel wall parameters. The short axial PDWI images were magnified by $200-300 \%$, and then the vessel area (VA) and lumen area (LA) at the most narrowed lumen (MNL) of the MCA and the reference site were measured manually. The reference site was defined as the nearest plaque-free or minimally diseased segments proximal to the stenotic site of the MCA. When a proximal reference site was not available, the neighbouring distal site was used.

Wall area $(\mathrm{WA})=\mathrm{VA}-\mathrm{LA}$, the plaque area $(\mathrm{PA})=$ WAMNL - WAreference, and the remodelling index (RI) $=$ VAMNL/VAreference. Normalised wall index (NWI) $=\mathrm{WA} / \mathrm{VA}$. The stenotic degree of the MCA was assessed using the following formula: (1 - LAMNL/VAreference) $\times 100 \%$. The parameter calculations were based on those in previous studies $(1,6,11)$.

Patients with hyperintense signals on DWI and hypointense signals on the apparent diffusion coefficient in attribution of the stenotic ipsilateral MCA were considered to have acute stroke, and were assigned to the symptomatic group. The other patients were assigned to the asymptomatic group.

$\mathrm{RI} \geq 1.05$ was defined as PR (Figure 1); RI 0.96-1.04 was defined as non-remodelling; $\mathrm{RI} \leq 0.95$ was defined as NR (Figure 2). The plaque contrast enhancement grade was subjectively categorised as follows: grade $0-$ the enhancement was less than or equal to that of normal intracranial arterial walls in the same patient; grade 1 - the enhancement was greater than that of normal intracranial arterial walls but less than that of the pituitary infundibulum; and grade 2-the enhancement was greater than or equal to that of the pituitary infundibulum (17).

Measurements were carried out by two experienced radiologists (observers D Zhang, X Yin), who had no knowledge of the patients' clinical data. The average values were calculated and used. The two observers agreed upon the degree of plaque enhancement and the slice for measurement. If disagreement arose between the observers, a consensus was reached through a joint reading.

\section{Statistical analysis}

All statistical analyses were performed using the IBM SPSS 21.0 package (Chicago, IL, USA). The normality of data was evaluated by conducting the Shapiro-Wilk test. The $t$-test was then applied for quantitative data between the two groups, and the chi-square test was used for categorical variables. Following this, the statistically significant indicators were analysed by both univariate and multivariate logistic regression analyses. $\mathrm{P}<0.05$ was deemed to represent statistical significance. Interobserver coherence between measurements was evaluated by intraclass correlation coefficient (ICC), with a two-way random effect for interobserver continuous variables. An ICC value of more than 0.75 showed strong interobserver reproducibility.

\section{Results}

\section{Demographic and clinical characteristics}

During the study period, 162 patients underwent MR examination. Among them, 97 patients were excluded for 

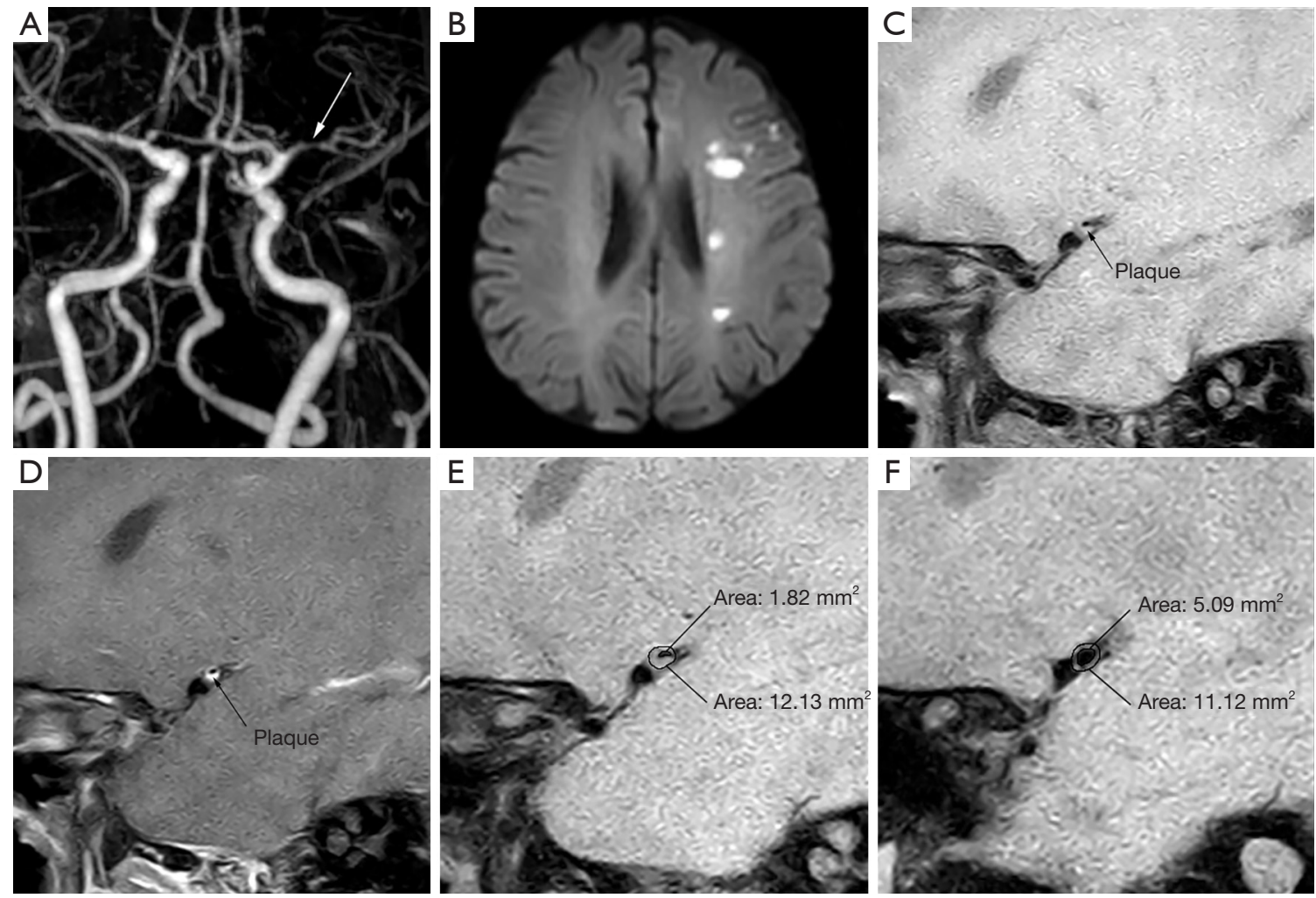

Figure 1 HRMRI of a patient with PR in the stenotic MCA. (A) The stenotic site of the left MCA. The white arrow shows the stenotic site of the left MCA; (B) acute ischemic stroke in the distribution of the left MCA; (C) T1-weighted HRMRI showing the plaque; (D) the plaque shows obvious enhancement in enhanced T1-weighted HRMRI; (E) PDWI showing the measurement at the most narrowed site. VA =12.13 $\mathrm{mm}^{2}, \mathrm{LA}=1.82 \mathrm{~mm}^{2}$; (F) measurement at the reference site in PDWI. VA $=11.12 \mathrm{~mm}^{2}, \mathrm{LA}=5.09 \mathrm{~mm}^{2}$. HRMRI, high-resolution magnetic resonance imaging; PR, positive remodelling; MCA, middle cerebral artery; PDWI, proton density weighted image; VA, vessel area; LA, lumen area.

the following reasons: moving artifacts $(n=35)$, ipsilateral ICA stenosis $\geq 50 \%(\mathrm{n}=41)$, brain tumour $(\mathrm{n}=5)$, and brain haemorrhage ( $\mathrm{n}=16)$ (Figure 3). Finally, 65 patients were enrolled into our prospective study. There were 30 patients in the symptomatic group ( 22 men, 8 women; mean age, $67 \pm 12$ years), and 35 patients in the asymptomatic group (28 men, 7 women; mean age, $69 \pm 14$ years). The two groups showed no significant difference in sex, alcoholism, hypertension, smoking, diabetes, age, and the levels of HbA1c, blood glucose, LDL, HDL, phospholipase-A2, triglycerides, total cholesterol, homocysteine, or white blood cell count (Table 1).

\section{Quantitative and qualitative variables of the MCA wall in the symptomatic and asymptomatic groups}

The interobserver reproducibility for the manual measurements and the evaluation of enhancement was strong, with an ICC of $0.913-0.937(\mathrm{P}<0.001)$. The quantitative and qualitative parameters of the symptomatic and asymptomatic groups are detailed in Tables 2,3. In the symptomatic group, the LAMNL $(\mathrm{P}=0.027)$ and LAreference $(\mathrm{P}=0.025)$ were smaller, and the NWIMNL $(\mathrm{P}=0.016)$, $\mathrm{PA}$ $(\mathrm{P}<0.001)$, and remodelling index $(\mathrm{P}<0.001)$ in the stenotic MCA were larger than those in the asymptomatic group. Compared to the asymptomatic group, the symptomatic group also had more superior/posterior plaques $(\mathrm{P}=0.001)$, a larger number of $\mathrm{PR}$ patterns $(\mathrm{P}<0.001)$, and more obviously enhanced plaques $(\mathrm{P}<0.001)$ (Tables 2,3).

\section{Univariate and multivariate logistic regression analyses for plaque characteristics}

Univariate logistic regression analysis showed that the PA, remodelling patterns, LAMNL, enhancement degree, and plaque location were predictors of acute stroke. Multivariate 

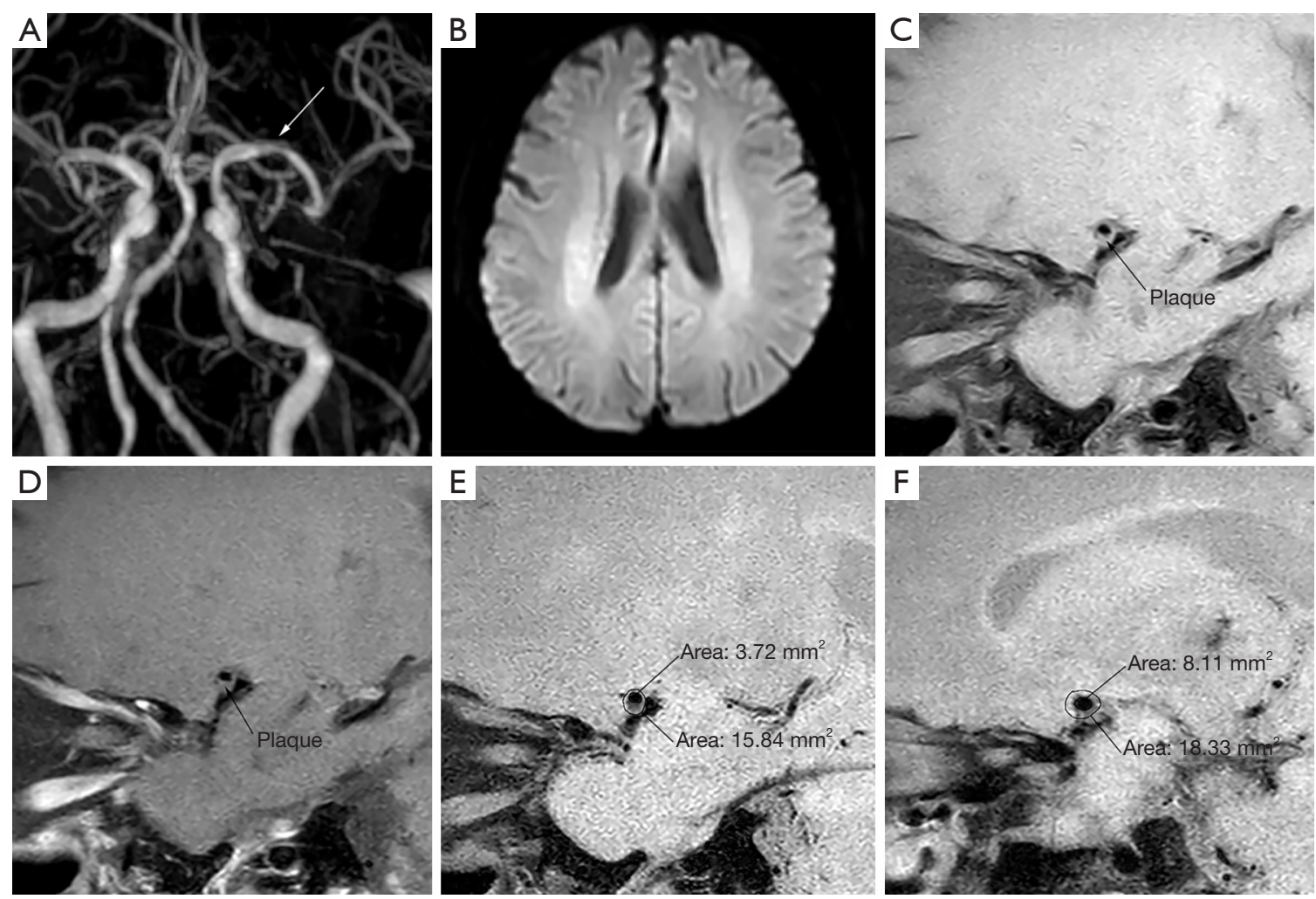

Figure 2 HRMRI of a patient with NR in the stenotic MCA. (A) The stenotic site of the left MCA. The white arrow shows the stenotic site of the left MCA; (B) DWI is normal; (C) T1-weighted HRMRI showing the plaque; (D) The plaque shows no enhancement in enhanced T1-weighted HRMRI; (E) PDWI showing the measurement at the most narrowed site in PDWI. VA =15.84 $\mathrm{mm}^{2}, \mathrm{LA}=3.72 \mathrm{~mm}^{2} ;(\mathrm{F})$ measurement at the reference site in PDWI. VA $=18.33 \mathrm{~mm}^{2}, \mathrm{LA}=8.11 \mathrm{~mm}^{2}$. HRMRI, high-resolution magnetic resonance imaging; NR, negative remodelling; MCA, middle cerebral artery; PDWI, proton density weighted image; VA, vessel area; LA, lumen area.

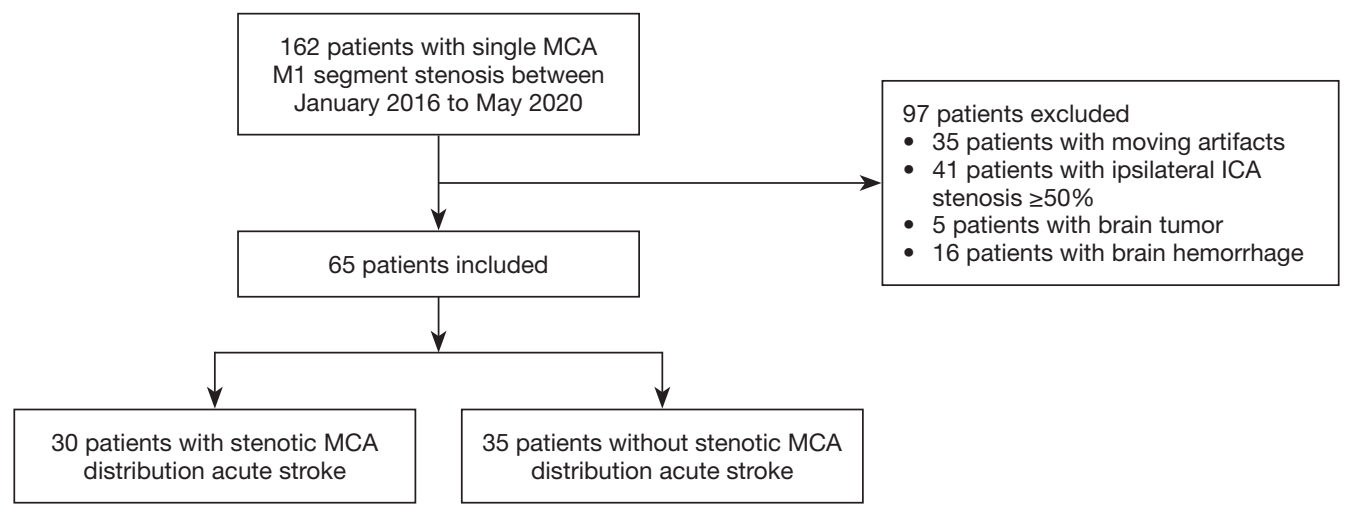

Figure 3 The flow chart for the study. MCA, middle cerebral artery; ICA, internal carotid artery.

logistic regression analysis showed that the combination of PA, LAMNL, and plaque enhancement degree had optimal predictive value (area under the curve $=0.927, \mathrm{P}<0.001$ ) (Table 4 and Figure 4).

\section{Discussion}

In this study, patients with atherosclerotic MCA stenosis in the symptomatic group had a smaller LAMNL, larger PA, higher RI, more superior/posterior plaques, more obviously 
Table 1 Demographic and clinical characteristics of symptomatic and asymptomatic patients

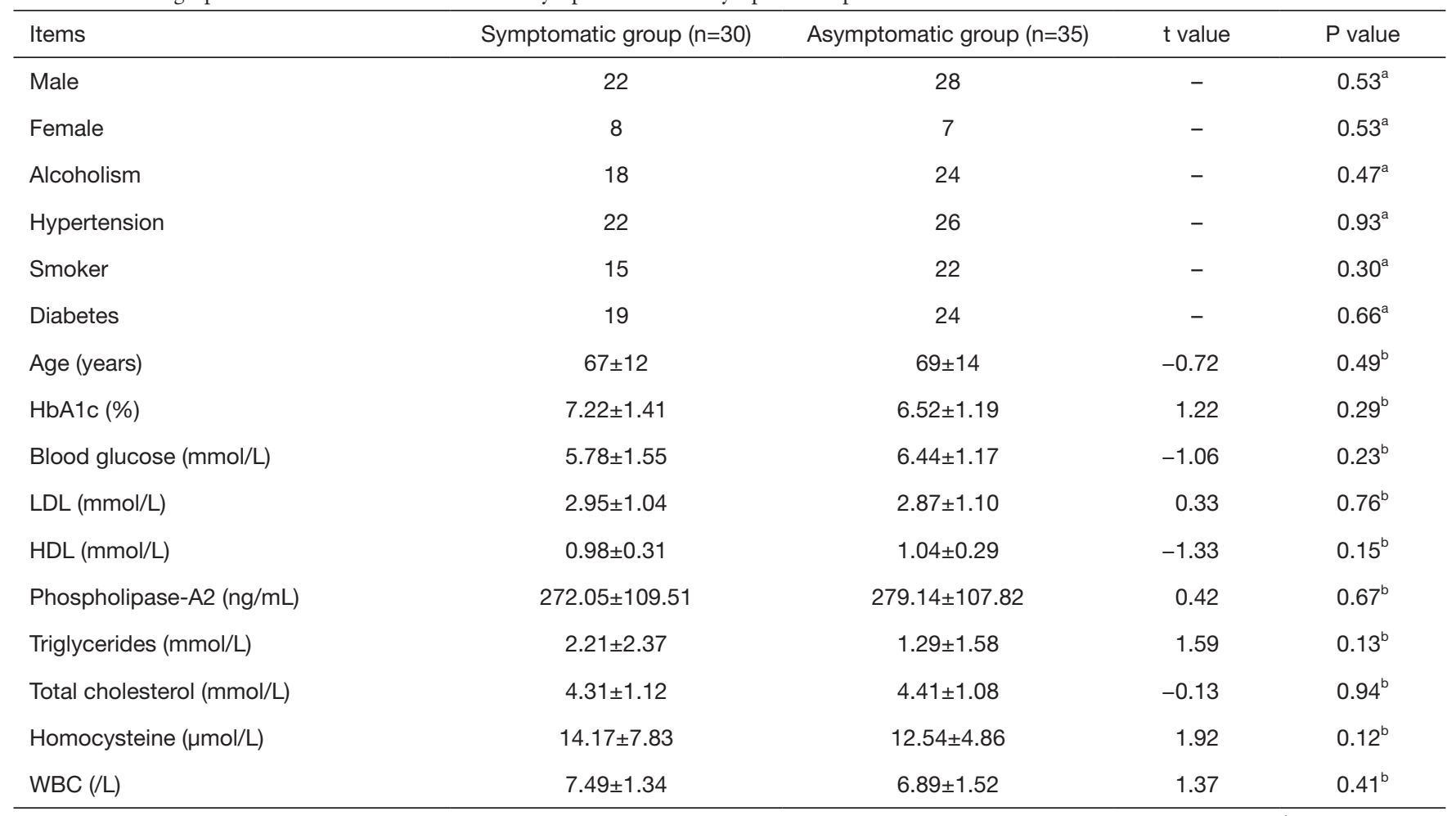

Data are presented as number or mean \pm standard deviation. ${ }^{a}, \mathrm{P}$ value was obtained by using Pearson's chi-square test; ${ }^{b}, \mathrm{P}$ value was obtained by $t$-test. LDL, low-density lipoprotein; HDL, high-density lipoprotein; WBC, white blood cell count.

Table 2 Quantitative data of the MCA wall in symptomatic group and asymptomatic group

\begin{tabular}{|c|c|c|c|c|}
\hline Items & Symptomatic group $(n=30)$ & Asymptomatic group $(n=35)$ & $\mathrm{t}$ value & $P$ value \\
\hline $\mathrm{VA}_{\mathrm{MNL}}\left(\mathrm{mm}^{2}\right)$ & $14.62 \pm 4.11$ & $14.00 \pm 2.93$ & 0.70 & 0.484 \\
\hline $\mathrm{LA}_{\mathrm{MNL}}\left(\mathrm{mm}^{2}\right)$ & $2.36 \pm 1.09$ & $2.96 \pm 1.01$ & -2.27 & $0.027^{*}$ \\
\hline Wall area $\mathrm{MNL}\left(\mathrm{mm}^{2}\right)$ & $12.26 \pm 3.98$ & $11.05 \pm 2.39$ & 1.51 & 0.136 \\
\hline $\mathrm{NWI}_{\mathrm{MNL}}$ & $0.83 \pm 0.08$ & $0.79 \pm 0.05$ & -2.46 & $0.016^{*}$ \\
\hline $\mathrm{LA}_{\text {reference }}\left(\mathrm{mm}^{2}\right)$ & $5.72 \pm 1.83$ & $6.79 \pm 1.88$ & -2.30 & $0.025^{*}$ \\
\hline $\mathrm{WA}_{\text {reference }}\left(\mathrm{mm}^{2}\right)$ & $7.83 \pm 2.93$ & $8.37 \pm 2.53$ & -0.80 & 0.428 \\
\hline$N W I_{\text {reference }}$ & $0.57 \pm 0.13$ & $0.55 \pm 0.08$ & -0.63 & 0.530 \\
\hline Degree of stenosis (\%) & $59.03 \pm 10.11$ & $57.00 \pm 7.85$ & 0.91 & 0.365 \\
\hline
\end{tabular}

Data are presented as mean \pm standard deviation. Data were contrasted using $t$-tests. ${ }^{*}, \mathrm{P}$ value $<0.05$. MCA, middle cerebral artery; VA, vessel area; MNL, most narrowed lumen; LA, lumen area; reference, reference site; WA, wall area; NWI, normal wall index; PA, plaque area; RI, remodelling index. 
Table 3 Qualitative data of the MCA wall in symptomatic group and asymptomatic group

\begin{tabular}{lccc}
\hline Items & Symptomatic group $(\mathrm{n}=30)$ & Asymptomatic group $(\mathrm{n}=35)$ & $\mathrm{P}$ value \\
\hline Superior plaques & 6 & 17 & $0.069^{\mathrm{a}}$ \\
Inferior plaques & 11 & 15 & 0.334 \\
Anterior plaques & 6 & 2 & 0.089 \\
Posterior plaques & 7 & 3 & 0.091 \\
Superior/posterior plaques & 13 & 32 & $0.001^{*}$ \\
Anterior/inferior plaques & 17 & 4 & $0.001^{*}$ \\
Obvious enhancement & 19 & 8 & $<0.001^{*}$ \\
Slight enhancement & 6 & 23 & 0.780 \\
No enhancement & 5 & 7 & $<0.001^{*}$ \\
PR & 24 & $<0.001^{*}$ &
\end{tabular}

a, $\mathrm{P}$ value was obtained by using corrected Pearson chi-square test. The other $\mathrm{P}$ values in the table were obtained by using Pearson's chi-square test; *, P value $<0.05$. MCA, middle cerebral artery; PR, positive remodelling.

Table 4 Univariate and multivariate logistic regression analyses showing the OR for plaque characteristics

\begin{tabular}{|c|c|c|c|c|}
\hline Items & OR value & $95 \% \mathrm{Cl}$ & $P$ value & AUC value \\
\hline Remodelling pattern & 16.00 & $4.73-54.15$ & $<0.001^{*}$ & 0.800 \\
\hline $\mathrm{LA}_{\mathrm{MNL}}\left(\mathrm{mm}^{2}\right)$ & 0.568 & $0.34-0.96$ & $0.033^{*}$ & 0.677 \\
\hline ED & 21.85 & $5.13-93.00$ & $<0.001^{\star}$ & 0.806 \\
\hline$P A+L A_{M N L}$ & 14.52 & $2.97-10.20$ & $<0.001^{*}$ & 0.825 \\
\hline$P A+L A_{M N L}+E D$ & 17.90 & $8.26-97.6$ & $<0.001^{*}$ & 0.927 \\
\hline
\end{tabular}

${ }^{*}, \mathrm{P}<0.05$. PA, plaque area; $L A_{M N L}$, lumen area in the most narrowed lumen; $E D$, enhancement degree.

enhanced plaques, and a larger number of PR pattern than those in the asymptomatic group (Figure 2). Moreover, logistic regression analysis showed that the combination of PA, LAMNL, and the plaque enhancement degree had optimal predictive value for acute stroke. Our study also showed that HRMRI is a suitable method for depicting the features of vessel walls and plaques clearly without exposing patients to radiation injury, and it has extensive applications in intracranial artery diseases.

As dynamic organs, arterial walls can change their morphology through the phenomenon of remodelling to compensate for lumen stenosis after plaque formation (18-20). Previous studies have identified two remodelling patterns: PR and NR. PR is considered to be an unsafe remodelling pattern that results in vessel enlargement; conversely, NR restricts the vessel, and is known as a relatively stable form of remodelling. Meanwhile, studies on coronary and carotid arteries reported that symptomatic patients had a higher RI and larger number of PR pattern than asymptomatic patients (19-21). Our study results were consistent with the above previous research findings. It is conceivable that the effect of PR on vessel enlargement increases the PA in the stenotic MCA. In line with this, the symptomatic group in our study had a larger PA and a smaller LAMNL than the asymptomatic group. Some scholars have reported similar results $(6,11,22)$. However, in our study, the symptomatic group had a smaller LA in the reference slice of the MCA than the asymptomatic group, although the NWI at the reference site did not show a significant difference. Therefore, after compensatory 

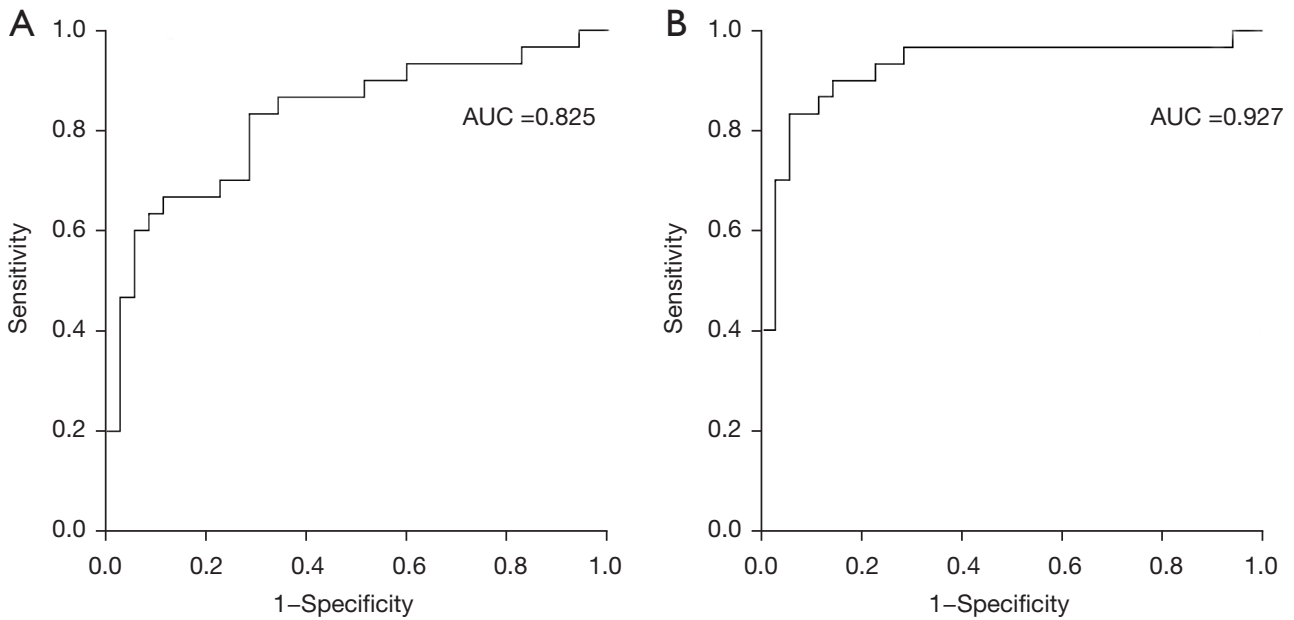

Figure 4 ROC curves for using PA + LAMNL (A) and PA + LAMNL + ED (B) to determine atherosclerotic plaques responsible for acute stroke. ROC, receiver operating characteristic; PA, plaque area; LAMNL, lumen area in the most narrowed lumen; ED, enhanced degree.

changes based on the NWI at the reference site the vascular wall parameters were in agreement. As previously reported, the NWI increases the comparability of vascular wall features between different patients and vessel areas, because it can avoid the difference in the WA caused by variation in vessel wall thickness $(23,24)$. However, many previous studies did not use the NWI for correction, which is an advantage of our study.

In recent years, some investigations have researched the clinical value of plaque enhancement in intracranial artery stenosis. Their results showed that the plaque enhancement identified on HRMRI could potentially serve as a marker of culprit plaques $(6,13,17)$. Pathological studies have arrived at similar conclusions. In our study, the symptomatic group had more obvious plaque enhancement than the asymptomatic group, which was the same as previous studies' findings. Zhao et al. $(25,26)$ suggested that patients with superior wall plaques were more likely to be symptomatic, possibly because of the proximity of these plaques to the orifices of penetrating arteries. Some penetrating arteries arise from the posterior wall of the MCA. Therefore, plaques located on the superior/ posterior side are more prone to induce acute stroke than those on the anterior/inferior vessel wall. In our study, the symptomatic group had a larger number of superior/ posterior plaques than the asymptomatic group, which was in agreement with previous studies (25-27).

In our research, the PA, remodelling pattern, LAMNL, enhancement degree, and plaque location were analysed between the symptomatic group and the asymptomatic group. Although there have been many studies $(1,2,6,7,9)$ about intracranial atherosclerotic plaques, few studies (6) have comprehensively discussed these risk factors. We also we performed univariate and multivariate logistic regression analyses for the above factors. We found that the combination of PA, LAMNL, and plaque enhancement degree had optimal predictive value for acute stroke. In summary, patients with plaques exhibiting a larger PA, smaller LAMNL, and obvious enhancement should actively seek therapeutic intervention, before the appearance of an acute stroke.

There were several limitations to our study. First, it was a prospective study with a limited number of patients. Second, we did not enroll patients with mild stenosis $(<30 \%)$ in the MCA M1 segment, which means that our results are not reflective of all patients with MCA stenosis. Third, our study lacked pathological information on plaques. Fourth, the calculation of the RI depended on the reference vascular wall data. Our study chose a proximal or distal segment as a reference site, which could cause an error in the RI due to the natural tapering of the MCA. Fifth, the enhancement degree of atherosclerotic plaques was judged subjectively by two radiological observers, rather than being an absolute measurement. Sixth, this was a preliminary study lacking research about large stroke lesions. We will perform a more in-depth study by increasing the sample size and improving the research approach. Finally, there were significantly more men in our study than women, which might be due to the small sample size. The sex ratio should be balanced in future research. All the above factors should be taken into 
consideration in follow-up research. We will do our best to perform conclusive research in the future.

\section{Conclusions}

In summary, the PA, remodelling pattern, LAMNL, enhancement degree, and plaque location are predictors of acute stroke. The combination of PA, LAMNL, and the plaque enhancement degree has optimal predictive value for acute stroke. Patients with stroke risk factors should be treated actively.

\section{Acknowledgments}

Funding: This study was funded by the National Natural Science Foundation of China (grant No. 81901806).

\section{Footnote}

Reporting Checklist: The authors have completed the STAndards for the Reporting of Diagnostic accuracy studies (STARD) reporting checklist. Available at https://dx.doi. org/10.21037/qims-21-531

Conflicts of Interest: All authors have completed the ICMJE uniform disclosure form (available at https://dx.doi. org/10.21037/qims-21-531). The authors have no conflicts of interest to declare.

Ethical Statement: The authors are accountable for all aspects of the work in ensuring that questions relating to the accuracy or integrity of any part of the work are appropriately investigated and resolved. The study was conducted in accordance with the Declaration of Helsinki (as revised in 2013). Approval for this retrospective cohort study was granted by the Ethics Committee of Nanjing Medical University. All participants or their legal representatives signed a written informed consent.

Open Access Statement: This is an Open Access article distributed in accordance with the Creative Commons Attribution-NonCommercial-NoDerivs 4.0 International License (CC BY-NC-ND 4.0), which permits the noncommercial replication and distribution of the article with the strict proviso that no changes or edits are made and the original work is properly cited (including links to both the formal publication through the relevant DOI and the license). See: https://creativecommons.org/licenses/by-nc-nd/4.0/.

\section{References}

1. Zhang DF, Chen YC, Chen H, Zhang WD, Sun J, Mao CN, Su W, Wang P, Yin X. A High-Resolution MRI Study of Relationship between Remodeling Patterns and Ischemic Stroke in Patients with Atherosclerotic Middle Cerebral Artery Stenosis. Front Aging Neurosci 2017;9:140.

2. Yu YN, Liu MW, Villablanca JP, Li ML, Xu YY, Gao S, Feng F, Liebeskind DS, Scalzo F, Xu WH. Middle Cerebral Artery Plaque Hyperintensity on T2-Weighted Vessel Wall Imaging Is Associated with Ischemic Stroke. AJNR Am J Neuroradiol 2019;40:1886-92.

3. Choi EH, Lee H, Chung JW, Seo WK, Kim GM, Ki CS, Kim YC, Bang OY. Ring Finger Protein 213 Variant and Plaque Characteristics, Vascular Remodeling, and Hemodynamics in Patients With Intracranial Atherosclerotic Stroke: A High-Resolution Magnetic Resonance Imaging and Hemodynamic Study. J Am Heart Assoc 2019;8:e011996.

4. Fukuda K, Iihara K, Maruyama D, Yamada N, Ishibashi-Ueda H. Relationship between carotid artery remodeling and plaque vulnerability with $\mathrm{T} 1$-weighted magnetic resonance imaging. J Stroke Cerebrovasc Dis 2014;23:1462-70.

5. Bentzon JF, Otsuka F, Virmani R, Falk E. Mechanisms of plaque formation and rupture. Circ Res 2014;114:1852-66.

6. Teng Z, Peng W, Zhan Q, Zhang X, Liu Q, Chen S, Tian X, Chen L, Brown AJ, Graves MJ, Gillard JH, Lu J. An assessment on the incremental value of high-resolution magnetic resonance imaging to identify culprit plaques in atherosclerotic disease of the middle cerebral artery. Eur Radiol 2016;26:2206-14.

7. Wu F, Song H, Ma Q, Xiao J, Jiang T, Huang X, Bi X, Guo X, Li D, Yang Q, Ji X, Fan Z; WISP Investigators. Hyperintense Plaque on Intracranial Vessel Wall Magnetic Resonance Imaging as a Predictor of Artery-to-Artery Embolic Infarction. Stroke 2018;49:905-11.

8. Leng X, Wong KS, Liebeskind DS. Evaluating intracranial atherosclerosis rather than intracranial stenosis. Stroke 2014;45:645-51.

9. Dieleman N, Yang W, Abrigo JM, Chu WC, van der Kolk AG, Siero JC, Wong KS, Hendrikse J, Chen XY. Magnetic Resonance Imaging of Plaque Morphology, Burden, and Distribution in Patients With Symptomatic Middle Cerebral Artery Stenosis. Stroke 2016;47:1797-802.

10. Zhu T, Ren L, Zhang L, Shao Y, Wan L, Li Y, Liang D, Zheng H, Liu X, Zhang N. Comparison of plaque 
characteristics of small and large subcortical infarctions in the middle cerebral artery territory using high-resolution magnetic resonance vessel wall imaging. Quant Imaging Med Surg 2021;11:57-66.

11. Ran Y, Wang Y, Zhu M, Wu X, Malhotra A, Lei X, Zhang F, Wang X, Xie S, Zhou J, Zhu J, Cheng J, Zhu C. Higher Plaque Burden of Middle Cerebral Artery Is Associated With Recurrent Ischemic Stroke: A Quantitative Magnetic Resonance Imaging Study. Stroke 2020;51:659-62.

12. Liao S, Deng Z, Wang Y, Jiang T, Kang Z, Tan S, Shan Y, Zou Y, Lu Z. Different Mechanisms of Two Subtypes of Perforating Artery Infarct in the Middle Cerebral Artery Territory: A High-Resolution Magnetic Resonance Imaging Study. Front Neurol 2018;9:657.

13. Wang E, Shao S, Li S, Yan P, Xiang Y, Wang X, Li J, Wang G, Sun Q, Du Y. A High-Resolution MRI Study of the Relationship Between Plaque Enhancement and Ischemic Stroke Events in Patients With Intracranial Atherosclerotic Stenosis. Front Neurol 2019;9:1154.

14. Lee HN, Ryu CW, Yun SJ. Vessel-Wall Magnetic Resonance Imaging of Intracranial Atherosclerotic Plaque and Ischemic Stroke: A Systematic Review and MetaAnalysis. Front Neurol 2018;9:1032.

15. Shi Z, Li J, Zhao M, Peng W, Meddings Z, Jiang T, Liu Q, Teng Z, Lu J. Quantitative Histogram Analysis on Intracranial Atherosclerotic Plaques: A HighResolution Magnetic Resonance Imaging Study. Stroke 2020;51:2161-9.

16. Lu SS, Ge S, Su CQ, Xie J, Mao J, Shi HB, Hong XN. MRI of plaque characteristics and relationship with downstream perfusion and cerebral infarction in patients with symptomatic middle cerebral artery stenosis. J Magn Reson Imaging 2018;48:66-73.

17. Qiao Y, Zeiler SR, Mirbagheri S, Leigh R, Urrutia V, Wityk R, Wasserman BA. Intracranial plaque enhancement in patients with cerebrovascular events on high-spatial-resolution MR images. Radiology 2014;271:534-42.

18. Glagov S, Weisenberg E, Zarins CK, Stankunavicius R, Kolettis GJ. Compensatory enlargement of human atherosclerotic coronary arteries. N Engl J Med 1987;316:1371-5.

19. Schoenhagen P, Ziada KM, Kapadia SR, Crowe TD, Nissen SE, Tuzcu EM. Extent and direction of arterial remodeling in stable versus unstable coronary syndromes : an intravascular ultrasound study. Circulation 2000;101:598-603.

20. Miao C, Chen S, Macedo R, Lai S, Liu K, Li D, Wasserman BA, Vogel-Claussen J, Lima JA, Bluemke DA. Positive remodeling of the coronary arteries detected by magnetic resonance imaging in an asymptomatic population: MESA (Multi-Ethnic Study of Atherosclerosis). J Am Coll Cardiol 2009;53:1708-15.

21. Hardie AD, Kramer CM, Raghavan P, Baskurt E, Nandalur KR. The impact of expansive arterial remodeling on clinical presentation in carotid artery disease: a multidetector CT angiography study. AJNR Am J Neuroradiol 2007;28:1067-70.

22. Shi MC, Wang SC, Zhou HW, Xing YQ, Cheng YH, Feng JC, Wu J. Compensatory remodeling in symptomatic middle cerebral artery atherosclerotic stenosis: a highresolution MRI and microemboli monitoring study. Neurol Res 2012;34:153-8.

23. Jia Q, Liu H, Li Y, Wang X, Jia J, Li Y. Combination of Magnetic Resonance Angiography and Computational Fluid Dynamics May Predict the Risk of Stroke in Patients with Asymptomatic Carotid Plaques. Med Sci Monit 2017;23:479-88.

24. Zhao X, Miller ZE, Yuan C. Atherosclerotic plaque imaging by carotid MRI. Curr Cardiol Rep 2009;11:70-7.

25. Zhao DL, Deng G, Xie B, Ju S, Yang M, Chen XH, Teng GJ. High-resolution MRI of the vessel wall in patients with symptomatic atherosclerotic stenosis of the middle cerebral artery. J Clin Neurosci 2015;22:700-4.

26. Zhao DL, Deng G, Xie B, Gao B, Peng CY, Nie F, Yang M, Ju S, Teng GJ. Wall characteristics and mechanisms of ischaemic stroke in patients with atherosclerotic middle cerebral artery stenosis: a high-resolution MRI study. Neurol Res 2016;38:606-13.

27. Sui B, Gao P, Lin Y, Jing L, Qin H. Distribution and features of middle cerebral artery atherosclerotic plaques in symptomatic patients: a 3.0T high-resolution MRI study. Neurol Res 2015;37:391-6.

Cite this article as: Zhang $\mathrm{D}$, Wang $M, \mathrm{Wu} \mathrm{L}$, Zhao $\mathrm{Y}$, Wang $\mathrm{S}$, Yin $\mathrm{X}, \mathrm{Wu} \mathrm{X}$. Assessing the characteristics and diagnostic value of plaques for patients with acute stroke using high-resolution magnetic resonance imaging. Quant Imaging Med Surg 2022;12(2):1529-1538. doi: 10.21037/qims-21-531 